\title{
ON GENERALIZED LEAST POWER APPROXIMATION
}

\author{
Nguyen Quang Dieu AND Phung VAN Manh*
}

Abstract. We study generalized least power approximation corresponding to certain sets of seminorms on Banach spaces. As applications, we construct sets of seminorms for trivariate harmonic polynomials and for Müntz polynomials such that the sequences of the generalized least power approximations converge uniformly.

Mathematics subject classification (2020): 41A10, 41A63, 41A65.

Keywords and phrases: Least power approximation, admissible meshes, trivariate harmonic polynomials, Müntz polynomials.

\section{REFERENCES}

[1] A. Axler, P. Bourdon, W. Ramey, Harmonic function theory, Springer-Verlag, New York, 2001.

[2] T. Bagby and N. Levenberg, Bernstein theorems for elliptic equations, J. Approx. Theory, 78 (1994), 190-212.

[3] D. Benko, T. ERdÉlyi, And J. Szabados, The full Markov-Newman inequalityfor Müntz polynomials on positive intervals, Proc. Amer. Math. Soc., 131 (2003), 2385-2391.

[4] P. BorWEIN AND T. ERdÉLYI, Newmann's inequality for Müntz polynomials on positive intervals, J. Approx. Theory, 85 (1996), 132-139.

[5] L. Bos, Fekete points as norming sets, Dolomites Res. Notes Approx., 11 (2018), 20-25.

[6] L. Bos, N. LEVENBERG, S. WALDRON, On the spacing of Fekete points for a sphere, ball or simplex, Indag. Mathem., N. S., 19 (2) (2008), 163-176.

[7] L. Bos, M. Vianello, Tchakaloff polynomial meshes, Ann. Polon. Math. 122 (2019), 221-231.

[8] L. Bos, S. De Marchi, A. Sommariva and M. Vianello, Computing multivariate Fekete and Leja points by numerical linear algebra, SIAM J. Numer. Anal., 40 (2010), 1948-1999.

[9] L. Bos, S. De Marchi, A. Sommariva And M. Vianello, Weakly admissible meshes and discrete extremal sets, Numer. Math. Theory Methods Appl., 4 (2011), 1-12.

[10] L. Bos AND M. Vianello, Low cardinality admissible meshes on quadrangles, triangles and disks, Math. Inequal. Appl., 15 (2012), 229-235.

[11] L. Bos, J.-P. Calvi, N. Levenberg, A. Sommariva And M. Vianello, Geometric weakly admissible meshes, discrete least square approximation and approximate Fekete points, Math. Comp, 90 (2011), 1623-1638.

[12] J.-P. CAlvi AND N. LeVEnBERG, Uniform approximation by discrete least square polynomials, J. Approx. Theory, 152 (2008), 82-100.

[13] M. VON GOLITSCHEK, Generalization of Jackson approximation theorems in the sense of Ch. Müntz, Bull. Amer. Math. Soc., 75 (1969), 524-528.

[14] A. KRoó, On optimal polynomial meshes, J. Approx. Theory, 163 (2011), 1107-1124.

[15] A. KRoó, Bernstein type inequalities on star-like domains in $\mathbb{R}^{d}$ with application to norming sets, Bull. Math. Sci., 3 (2013), 349-361.

[16] A. KRoó, On the existence of optimal meshes in every convex domain on the plane, J. Approx. Theory, 238 (2019), 26-37.

[17] D. Leviatan, On Jackson-Müntz theorem, J. Approx. Theory, 10 (1974), 1-5.

[18] J. Marzo, J. OrtegA-Cerdà, Equidistribution of Fekete points on the sphere, Constr. Approx., 32 (2010), 513-521.

[19] F. Piazzon and M. Vianello, Analytic transformations of admissible meshes, East J. Approx., 16 (2010), 389-398. 
[20] F. Piazzon, A. Sommariva And M. Vianello, Caratheodory-Tchakaloff Subsampling, Dolomites Res. Notes Approx., 10 (2017), 5-14.

[21] F. Piazzon and M. Vianello, Jacobi norming meshes, Math. Inequal. Appl. 19 (2016), 10891095.

[22] F. Piazzon and M. Vianello, Small pertubations of admissible meshes, Appl. Anal., 62 (2013), 1062-1073.

[23] F. Piazzon, Optimal polynomial admissible meshes on some classes of compact sets of $\mathbb{R}^{d}$, J. Approx. Theory, 207 (2016), 241-264.

[24] Phung V. M., Phan T. T. And Mai H. A., On generalized least square approximation, Dolomites Res. Notes Approx., 12 (2019), 101-110.

[25] T. J. RivLin, An introduction to the approximation of functions, Blaisdell Publishing Co. Ginn and Co., Waltham, Mass.-Toronto, Ont.-London, 1969.

[26] G. Szego, On the gradient of solid harmonic polynomials, Trans. Amer. Math. Soc., 47 (1940), $51-$ 65.

[27] M. Vianello, Compressed sampling inequalities by Tchakaloff's theorem, Math. Inequal. Appl., 19 (2016), 395-400. 